\title{
138.
}

\section{RECHERCHES SUR LES MATRICES DONT LES TERMES SONT DES FONCTIONS LINÉAIRES D'UNE SEULE INDÉTERMINÉE.}

[From the Journal für die reine und angewandte Mathematik (Crelle), tom. L. (1855), pp. $313-317$.

JE pose la matrice

$$
\left|\begin{array}{ccc}
A, & B, & C, \ldots \\
A^{\prime}, & B^{\prime}, & C^{\prime}, \ldots \\
A^{\prime \prime}, & B^{\prime \prime}, & C^{\prime \prime}, \ldots \\
\vdots & &
\end{array}\right|
$$

dont les termes ( $n^{2}$ en nombre) sont des fonctions linéaires d'une quantité $s$, et je considère le déterminant formé avec cette matrice, et les déterminants mineurs formés en supprimant un nombre quelconque des lignes et un nombre égal de colonnes de la matrice. En supprimant une seule ligne et une seule colonne, on obtient les premiers mineurs; en supprimant deux lignes et deux colonnes, on obtient les seconds mineurs; et ainsi de suite. Cela étant, je suppose que la quantité $s$ a été trouvée en égalant à zéro le déterminant formé avec la matrice donnée; ce déterminant sera une fonction de $s$ du $n$-ième degré qui généralement ne contiendra pas de facteurs multiples. On voit donc qu'un facteur simple du déterminant ne peut pas entrer comme facteur dans les premiers mineurs (c'est-à-dire dans tous les premiers mineurs); mais en supposant que le déterminant ait des facteurs multiples, un facteur multiple du déterminant peut entrer comme facteur (simple ou multiple) dans les premiers mineurs, ou dans les mineurs d'un ordre plus élevé. Il importe de trouver le degré selon lequel un facteur multiple du déterminant peut entrer comme facteur des premiers mineurs, u des mineurs d'un ordre quelconque donné.

Cela se fait très facilement au moyen d'une propriété générale des déterminants; si les mineurs du $(r+1)$ ième ordre contiennent le facteur $(s-a)^{a}$ (c'est-à-dire, si tous 
les mineurs de cet ordre contiennent le facteur $(s-a)^{a}$, mais non pas tous les facteurs $\left.(s-a)^{a+1}\right)$; et si de même les mineurs du $r$-ième ordre contiennent le facteur $(s-a)^{\beta}$; alors les mineurs du $(r-1)$ ième ordre contiendront au moins le facteur $(s-a)^{2 \beta-\alpha}$. Autrement dit: les mineurs du. $(r-1)$ ième ordre contiendront le facteur $(s-a)^{\gamma}$ où $\gamma>2 \beta-\alpha$, ou, ce qui est la même chose, $\alpha-2 \beta+\gamma \nless 0$; c'est-à-dire: en formant la suite des indices des puissances selon lesquelles le facteur $(s-a)$ entre dans les mineurs premiers, seconds, \&c. (il va sans dire que cette suite sera une suite décroissante), les différences secondes seront positives [c'est-à-dire non négatives]. Je représente par $\alpha, \beta, \gamma, \ldots$ la suite dont il s'agit; je suppose, pour fixer les idées, que $\delta$ soit le dernier terme qui ne s'évanouisse pas, et j'écris

$$
\begin{aligned}
& \alpha, \quad \beta, \quad \gamma, \quad \delta, \quad 0,0, \ldots \\
& \alpha-\beta, \quad \beta-\gamma, \quad \gamma-\delta, \quad \delta, \quad 0, \ldots \\
& \alpha-2 \beta+\gamma, \quad \beta-2 \gamma+\delta, \quad \gamma-2 \delta, \quad \delta, \quad 0, \ldots \text {; }
\end{aligned}
$$

ici, quel que soit le nombre des termes, tous les nombres de la troisième ligne seront positifs, et en représentant ces nombres par $\int, \int^{\prime}, \int^{\prime \prime}, \& c$., on obtient:

$$
\begin{array}{lr}
\alpha=\int+2 \int^{\prime}+3 f^{\prime \prime}+4 f^{\prime \prime \prime}+\ldots \\
\beta= & f^{\prime}+2 \int^{\prime \prime}+3 \int^{\prime \prime \prime}+\ldots \\
\gamma= & f^{\prime \prime}+2 f^{\prime \prime \prime}+\ldots \\
\delta= & f^{\prime \prime \prime}+\ldots
\end{array}
$$

Il y a ici à considérer que le nombre $\alpha$, indice de la puissance selon laquelle le facteur $(s-a)$ entre dans le déterminant, est donné; il sera donc permis de prendre. pour $\int, f^{\prime}, f^{\prime \prime}, \ldots$ des valeurs entières et positives quelconques (zéro y compris) qui satisfont à la première équation; les autres équations donnent alors les valeurs de $\beta, \gamma, \delta, \& c$. On forme de cette manière une table des particularités que peut présenter un facteur multiple $(s-a)^{a}$ du déterminant; cette table est composée des symboles $\alpha, \beta, \gamma, \ldots$, et les nombres $\alpha, \beta, \ldots$ de chaque symbole font voir le degré selon lequel le facteur $(s-a)$ entre dans les déterminants, dans les mineurs premiers, seconds, \&c. Or il est très facile de former, au moyen des tables pour $\alpha=1, \alpha=2, \ldots \alpha=k$, la table pour $\alpha=k+1$. On a par exemple pour $\alpha=1, \alpha=2, \alpha=3, a=4$ les tables suivantes:

$$
\begin{aligned}
& \text { Pour } \alpha=1, \quad 1 \text {. } \\
& \text { Pour } \alpha=2, \quad 2,21 . \\
& \text { Pour } \alpha=3, \quad 3,31,321 . \\
& \text { Pour } \alpha=4,4,41,42, \quad 421,4321 .
\end{aligned}
$$

De là on tire la table pour $\alpha=5$, savoir :

$$
\text { Pour } \alpha=5, \quad 5, \quad 51,52,521,531,5321,54321 .
$$

En effet, le premier terme est 5 , et on obtient les autres termes en mettant le nombre $\check{j}$ devant les symboles des tables pour $\alpha=1, \alpha=2, \alpha=3, \alpha=4$, en ayant seulement soin C. II. 
de supprimer les symboles 53,54,541, 542, 5421 pour lesquels le premier terme de la suite des différences secondes est négatif. On trouve de même pour $\alpha=6$, la table suivante, savoir :

Pour $\alpha=6,6,61,62,621,63,631,6321,642,6421,64321,654321$;

et ainsi de suite. Les nombres des symboles pour $\alpha=1,2,3,4,5,6,7,8$, \&c. sont $1,2,3,5,7,11,15,22,[30,42,56], \& c$; ; ce sont les coefficients des puissances $x^{1}, x^{2}, x^{3}$ \&c. dans le développement de

$$
(1-x)^{-1}\left(1-x^{2}\right)^{-1}\left(1-x^{3}\right)^{-1}\left(1-x^{4}\right)^{-1}\left(1-x^{5}\right)^{-1} \ldots \text { \&c. }
$$

fonctions qui se présentent, comme on sait, dans la théorie de la partition des nombres.

Maintenant, au lieu de considérer un seul facteur du déterminant, je considère tous les facteurs: par exemple pour $n=4$, le déterminant peut avoir un facteur double $(s-a)^{2}$, et un autre facteur double $(s-b)^{2}$; il peut de plus arriver que le facteur $(s-a)$ soit facteur simple des premiers mineurs, mais que le facteur $(s-b)$ n'entre pas dans les premiers mineurs. Le symbole qui correspond au facteur $(s-a)$ sera 21 , et le symbole qui correspond au facteur $(s-b)$ sera 2 . En combinant ces deux symboles, on aura le symbole composé $\begin{aligned} & 21 \\ & 2\end{aligned}$, qui dénote que le déterminant a deux facteurs doubles de

la classe dont il s'agit. Je forme de ces symboles composés des tables pour $n=1$, $n=2, n=3, n=4$, \&c. On a :

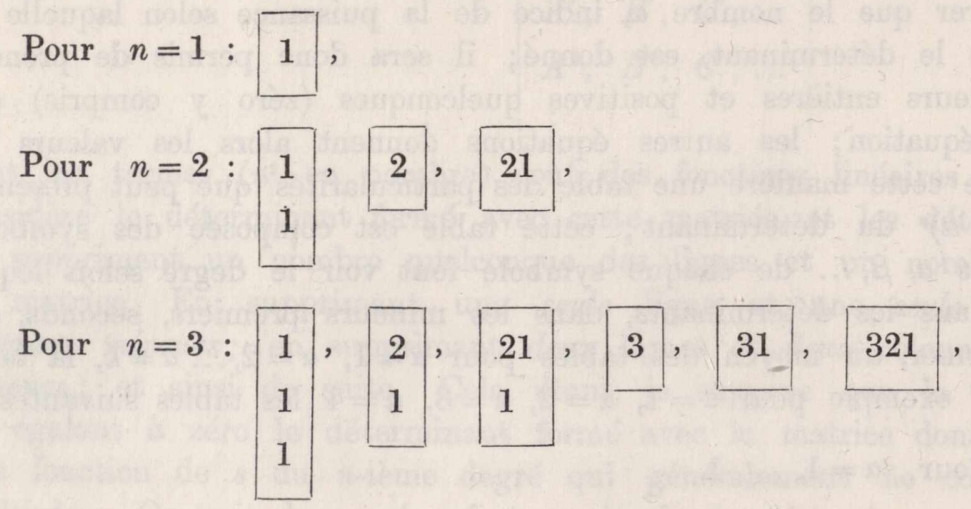

Pour $n=4$ :

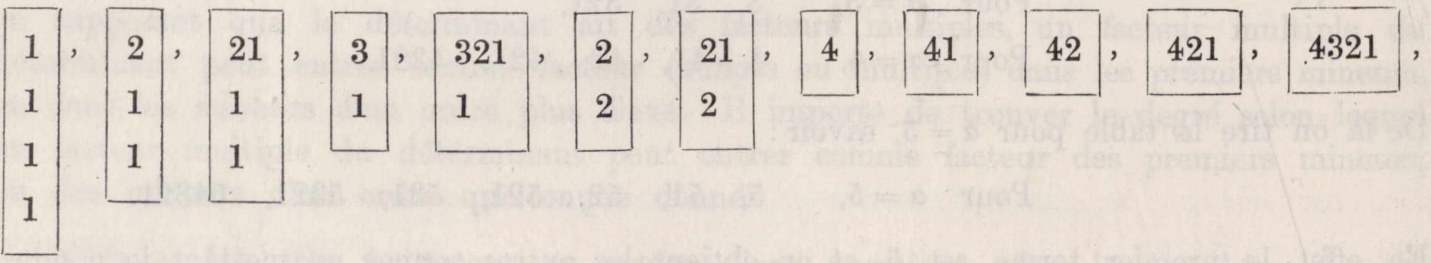

et ainsi de suite. 
Pour donner encore un exemple du sens de ces symboles, le symbole $\begin{aligned} & 321 \\ & 1\end{aligned} \mid$ dénote que le déterminant a un facteur $(s-a)$ qui entre comme facteur triple dans le déterminant, comme facteur double dans les premiers mineurs, et comme facteur simple dans les seconds mineurs; l'autre facteur du déterminant est un facteur simple $(s-b)$. Les nombres des symboles pour $n=1,2,3,4,5,6,7,8,9,10,11$, \&c. sont $1,3,6$, $14,27,58,111,223,424,817,1527$, \&c.; ces nombres sont les coefficients de $x^{1}, x^{2}, x^{3}$, \&c. dans le développement de

$$
(1-x)^{-1}\left(1-x^{2}\right)^{-2}\left(1-x^{3}\right)^{-3}\left(1-x^{4}\right)^{-5}\left(1-x^{5}\right)^{-7}\left(1-x^{6}\right)^{-11}\left(1-x^{7}\right)^{-15}\left(1-x^{8}\right)^{-22}\left(1-x^{9}\right)^{-30} \ldots \text { \&c. }
$$

où les indices $1,2,3,5,7,11, \&$ c. forment la suite qui se présente dans la théorie de la partition des nombres, dont j'ai parlé plus haut. Il est très facile de démontrer qu'il en est ainsi.

Les résultats que je viens de présenter sont en partie dus à M. Sylvester (voyez son mémoire "An enumeration of the contacts of lines and surfaces of the second order," Philosophical Magazine, [vol. I. (1851), pp. 18-20]). En effet, M. Sylvester commence par étendre à des fonctions d'un nombre quelconque d'indéterminées l'idée géométrique des contacts des courbes et des surfaces. En considérant les deux équations quadratiques $U=0, V=0$, il forme le discriminant de la fonction quadratique $U+s V$, et il cherche dans quel degré chaque facteur de ce discriminant peut entrer comme facteur dans les mineurs premiers, seconds, \&c. Le discriminant de M. Sylvester est un déterminant symétrique; mais cela ne change rien à la question, et je n'ai fait que reproduire l'analyse de M. Sylvester, en donnant cependant l'algorithme pour la formation des symboles, et de plus la loi pour le nombre des symboles. M. Sylvester donne pour $n=2,3,4,5,6$, des nombres qui, en ajoutant à chacun le nombre 2, pour embrasser deux cas extrêmes qui ne sont pas comptés, seraient $3,6,14,26,58$. Il se trouve dans le nombre 26 une erreur de calcul; ce nombre devrait être 27 , et en suppléant le premier terme 1 , on a la suite trouvée plus haut, savoir $1,3,6,14$, 27, 58, \&c.; il y a de même une erreur de calcul dans les nombres donnés par M. Sylvester pour $n=7$ et $n=8$.

Mais tout cela s'applique à une autre théorie géométrique, savoir à la théorie des figures homographes. Pour fixer les idées, je ne considère que les figures dans le plan. En supposant que $x, y, z$ soient les coordonnées d'un point, et en prenant pour (x, y, z) des fonctions linéaires de $(x, y, z)$ on aura $(\mathrm{x}, \mathrm{y}, \mathrm{z})$ comme coordonnées d'un point homographe au point $(x, y, z)$. En cherchant les points qui sont homographes chacun à soi-même, on est conduit aux équations $\mathrm{x}-s x=0, \mathrm{y}-s y=0, \quad \mathrm{z}-s z=0$. Les quantités à gauche $\mathrm{x}-s x, \mathrm{y}-s y, \mathrm{z}-s z$ sont des fonctions linéaires de $x, y, z$, ayant pour coefficients des fonctions linéaires de $s$. On a ainsi une matrice dont les termes sont des fonctions linéaires de $s$; la théorie entière se rattache aux propriétés de cette matrice. Pour le cas général de l'homographie ordinaire, on a le symbole $28-2$ 
1
1
1 , pour l'homologie, le symbole $\left[\begin{array}{l}21 \\ 1\end{array}\right]$; les autres symboles $\left[\begin{array}{l}2 \\ 1\end{array}\right],[3, \square 3$ se rapportent à des cas moins généraux, et le symbole 321 au cas de l'identité complète des deux figures; y compris ce cas-limite de l'identité complète, il existe pour le plan 6 espèces d'homographie; pour l'espace ordinaire il existe 14 espèces d'homographie. Je reviendrai à cette théorie à une autre occasion.

Londres, le 24 Mai 1854. 